\title{
Properties of Chemical and Kinetic Freeze-Outs in High-Energy Nuclear Collisions
}

\author{
Fu-Hu Liu (D), Sakina Fakhraddin, ${ }^{2,3}$ Roy A. Lacey, ${ }^{4}$ Raghunath Sahoo $\left(\mathbb{D},{ }^{5}\right.$ \\ Edward K. Sarkisyan-Grinbaum, ${ }^{6,7}$ and Bhartendu K. Singh ${ }^{8}$ \\ ${ }^{1}$ Institute of Theoretical Physics, Shanxi University, Taiyuan, Shanxi 030006, China \\ ${ }^{2}$ Physics Department, College of Science \& Arts in RK, Qassim University, Buraidah 51452, Qassim, Saudi Arabia \\ ${ }^{3}$ Physics Department, Faculty of Science, Sana'a University, P.O. Box 1247, Sana'a, Yemen \\ ${ }^{4}$ Departments of Chemistry \& Physics, Stony Brook University, Stony Brook, NY 11794, USA \\ ${ }^{5}$ Discipline of Physics, School of Basic Sciences, Indian Institute of Technology Indore, Indore 453552, India \\ ${ }^{6}$ Experimental Physics Department, CERN, 1211 Geneva 23, Switzerland \\ ${ }^{7}$ Department of Physics, The University of Texas at Arlington, Arlington, TX 76019, USA \\ ${ }^{8}$ Physics Department, Banaras Hindu University, Varanasi 221005, India \\ Correspondence should be addressed to Fu-Hu Liu; fuhuliu@163.com
}

Received 25 September 2018; Accepted 26 September 2018; Published 17 October 2018

Copyright (C) $2018 \mathrm{Fu}-\mathrm{Hu}$ Liu et al. This is an open access article distributed under the Creative Commons Attribution License, which permits unrestricted use, distribution, and reproduction in any medium, provided the original work is properly cited. The publication of this article was funded by $\mathrm{SCOAP}^{3}$.

The search for a new state of matter and understanding of the conditions of its formation in the extreme conditions of the dense and high-temperature environment is the main goal of the ongoing heavy ion investigations at the CERN LHC experiments such as ALICE, ATLAS, and CMS where heavy-ions are smashed at unprecedented high energies ever reached. The study of these high-energy nuclear collisions is also believed to approach the conditions close to those at the formation of the early Universe which has been undergoing under extreme dense and temperature. The understanding of the formed nuclear matter is a main topic of the today's most theoretical and phenomenological models aimed to describe the conditions and to find the equation of state of the matter formed in heavy ion collisions at the LHC believed to be the quark-gluon plasma, predicted by the theory of strong interaction Quantum Chromodynamics (QCD) being currently under extensive development. The understanding of the features observed in nuclear collisions at high energies at the LHC and at RHIC is also believed to provide keys explaining the observations in nuclear collisions at lower energies of the studies planned and ongoing worldwide as well as unexpected nuclear-type findings in proton-proton collisions at LHC.
To clarify the above points it is crucial to understand the space-time evolution of the system formed during the collision and resulted into the observed particles, mostly hadrons. In these studies, one distinguishes the two so-called freeze-outs, namely, the chemical freeze-out and the kinetic freeze-out, so that the momentum spectra of produced particles are frozen in time. The understanding of this picture is still under wide discussion and attracts lot of interest, as on the one hand, one assumes the chemical and kinetic freezeouts to occur without any time lag, while on the other hand, there are models considering time interval so that the kinetic freeze-out comes after the chemical one.

This special issue concerns many topics on properties of chemical and kinetic freeze-outs in high-energy nuclear (and particle) collisions, for example, (i) describing particle distributions and correlations and studying statistical laws and dynamical properties related to the special topic; (ii) extracting thermal and dynamical properties of interacting system and formed matters and real thermodynamic parameters of chemical and kinetic freeze-outs; (iii) extracting radial flow velocity and describing elliptic flow and other anisotropic flow; (iv) searching for the softest points of the equation of state and the critical point of hadronic matter to 
quark-gluon plasma; and (v) studying collective behavior in small system.

In the article "Chiral Phase Transition in Linear Sigma Model with Nonextensive Statistical Mechanics," K.-M. Shen et al. investigate the chiral phase transition at finite temperature and baryon chemical potential in the framework of the linear sigma model. The corresponding nonextensive distribution is characterized by a nonextensive parameter and the results in the usual Boltzmann-Gibbs case are recovered when the nonextensive parameter levels off to one. The thermodynamics of the linear sigma model and its corresponding phase diagram are analyzed. Some behaviors of the critical temperature, chemical potential, and nonextensive parameter are studied in the phase diagram.

In the article "Mean Field Approximation for the Dense Charged Drop," S. Bondarenko and K. Komoshvili consider the mean field approximation for the description of the probe charged particle in a dense charged drop, solve the corresponding Schrödinger equation for the drop with spherical symmetry in the first order of mean field approximation, and discuss the obtained results. This work is useful for the clarification of the spectrum of the produced particles, which is influenced by the quantum-mechanical properties of the QCD fireball. This approach also provides the connection between the data obtained in the LHC and RHIC experiments and microscopic fields inside the collision region.

In the article "A Comparative Study of $K^{ \pm} / \pi^{ \pm}$Ratio in Proton-Proton Collisions at Different Energies: Experimental Results versus Model Simulation," S. Bhattacharyya et al. present a systematic study of $K^{+} / \pi^{+}, K^{-} / \pi^{-}$, and $\left(K^{+}+\right.$ $\left.K^{-}\right) /\left(\pi^{+}+\pi^{-}\right)$ratio in proton-proton collisions as a function of the bombarding energy from $6.3 \mathrm{GeV}$ to $7 \mathrm{TeV}$ using the UrQMD model and DPMJET III model. Comparisons of the simulated results with the available experimental data are also presented. Dependence of $K^{+} / \pi^{+}$and $K^{-} / \pi^{-}$ on energy shows different behaviors for the UrQMD and DPMJET III models. The presence of the horn-like structure in the variation of $K^{+} / \pi^{+}$and $K^{-} / \pi^{-}$with energy for the experimental data is supported by the DPMJET III model.

In the article "A Description of Pseudorapidity Distributions of Charged Particles Produced in $\mathrm{Au}+\mathrm{Au}$ Collisions at RHIC Energies," Z. J. Jiang et al. assume that the hot and dense matter expands according to the hydrodynamic model including phase transition and decouples into particles via the prescription of Cooper-Frye. The leading particles are as usual supposed to have Gaussian rapidity distributions with the number equaling that of participants. This investigation shows that the leading particles are essential in describing the pseudorapidity distributions of charged particles produced in high energy heavy ion collisions. This might be due to the different transparencies of nuclei at different energies.

In the article "Azimuthal Anisotropy in High-Energy Nuclear Collision: An Approach Based on Complex Network Analysis," S. Bhaduri and D. Ghosh attempt to find deterministic information on the anisotropy in azimuthal distribution by means of precise determination of topological parameter from a complex network perspective. They construct visibility graphs from their corresponding azimuthal space or $\phi$-data sets. From each visibility graph, a number of cluster data sets are extracted, and then again visibility graphs are constructed for each cluster data set and its Monte Carlo simulated counterpart. Each of these clusters is self-similar and scalefree and is of fractal structure.

The article "Comparing Standard Distribution and Its Tsallis Form of Transverse Momenta in High Energy Collisions" investigates the experimental (simulated) transverse momentum spectra of $\pi^{-}$produced at midrapidity in central nucleus-nucleus collisions at the SIS, RHIC, and LHC energies obtained by different collaborations, where a few simulated data are taken from the results of FOPI Collaboration which uses the IQMD transport code based on Quantum Molecular Dynamics. A two-component standard distribution and the Tsallis form of standard distribution are used to fit these data in the framework of a multisource thermal model. The excitation functions of main parameters in the two distributions are analyzed.

The article "Renormalization Group Equation for Tsallis Statistics" investigates the scaling properties of thermofractals through the renormalization group equation, known as Callan-Symanzik equation. The Callan-Symanzik equation associated with Tsallis statistics is derived in this article in association with the thermofractal scale-free structure, setting new grounds for the interpretation of nonextensive thermodynamics in terms of renormalization group theory and opening new possibilities of its application in QCD related problems. In addition, this article can be also associated with the nonthermal phase transition of hadronic matter associated with quark-gluon plasma.

The article "A Description of the Transverse Momentum Distributions of Charged Particles Produced in Heavy Ion Collisions at RHIC and LHC Energies" uses nonextensive statistics together with relativistic hydrodynamics including phase transition to discuss the transverse momentum distributions of charged particles produced in heavy ion collisions, by assuming the existence of memory effects and long-range interactions. The combined contributions from nonextensive statistics and hydrodynamics can give a good description of the experimental data in $\mathrm{Au}+\mathrm{Au}$ collisions at $200 \mathrm{GeV}$ and in $\mathrm{Pb}+\mathrm{Pb}$ collisions at $2.76 \mathrm{TeV}$ for $\pi^{ \pm}$and $K^{ \pm}$in wide transverse momentum region and for $p(\bar{p})$ in narrow transverse momentum region.

The article "Correlations and Event-by-Event Fluctuations in High Multiplicity Events Produced in ${ }^{208} \mathrm{~Pb}-{ }^{208} \mathrm{~Pb}$ Collisions" analyzes high multiplicity events produced in $158 \mathrm{~A} \mathrm{GeV} / \mathrm{c}{ }^{208} \mathrm{~Pb}^{208} \mathrm{~Pb}$ collisions to study the event-byevent fluctuations. The findings reveal that the method of scaled factorial moments can be used to identify the events having densely populated narrow phase space bins. A few events sorted out by adopting this approach are individually analyzed. It is observed that these events do exhibit large fluctuations in their pseudorapidity and azimuthal angle distributions arising due to some dynamical reasons. Some complex two-dimensional structures render some dynamical origins.

In the article "Entropy and Multifractality in Relativistic Ion-Ion Collisions," S. Khan and S. Ahmad investigate entropy production in multiparticle systems by analyzing the experimental data on ion-ion collisions at AGS and SPS 
energies and comparing the findings with those reported earlier for hadron-hadron, hadron-nucleus, and nucleusnucleus collisions. It is observed that the entropy produced in limited and full phase space, when normalized to maximum rapidity, exhibits a kind of scaling which is nicely supported by Monte Carlo model HIJING. The findings reveal that Rényi's order information entropy could be another way to investigate the fluctuations in multiplicity distributions in terms of spectral function.

In the article "Radial Flow in a Multiphase Transport Model at FAIR Energies," S. Sarkar et al. compare azimuthal distributions of radial velocities of charged hadrons produced in nucleus-nucleus collisions with the corresponding azimuthal distribution of charged hadron multiplicity in the framework of a multiphase transport (AMPT) model at two different collision energies. The mean radial velocity seems to be a good probe for studying radial expansion. While the anisotropic parts of the distributions indicate a kind of collective nature in the radial expansion of the intermediate "fireball" and their isotropic parts characterize a thermal motion, these simulation results at FAIR are also interesting to compare with those at RHIC and LHC.

In the article "Multifractal Analysis of Charged Particle Multiplicity Distribution in the Framework of Renyi Entropy," S. Bhattacharyya et al. present an analysis of multifractality and multifractal specific heat in the frame work of Rényi entropy analysis for the produced shower particles in ${ }^{16} \mathrm{O}-\mathrm{AgBr},{ }^{28} \mathrm{Si}-\mathrm{AgBr}$, and ${ }^{32} \mathrm{~S}-\mathrm{AgBr}$ interactions at $4.5 \mathrm{~A}$ $\mathrm{GeV} / \mathrm{c}$. Experimental results are compared with the prediction of the UrQMD model. Qualitative information about the multifractal dynamics of particle production process are extracted and reported. The investigation of quark-hadron phase transition in the mentioned interactions in the framework of Ginzburg-Landau theory are also presented.

The article "HBT Radii: Comparative Studies on Collision Systems and Beam Energies" reviews shortly the experimental results on the two-particle Hanbury-Brown-Twiss (HBT) interferometry which is an important probe for understanding the space-time structure of particle emission sources in high energy heavy ion collisions. The HBT radii in central $\mathrm{Pb}+\mathrm{Pb}$ collisions at $17.3 \mathrm{GeV}$, in central $\mathrm{Au}+\mathrm{Au}$ collisions at 19.6 GeV, and in central $\mathrm{Cu}+\mathrm{Cu}$ collisions at $22.4 \mathrm{GeV}$ are compared with each other. A similarity in the $R_{\text {out }} / R_{\text {side }}$ ratio with transverse mass across the collision systems is found at the energy bridge from the SPS to RHIC. A rise of the $R_{\text {out }} / R_{\text {side }}$ ratio at this energy bridge is not observed.

The article "Angular Dependence of $\eta$ Photoproduction in Photon-induced Reaction" studies the angular dependence of $\eta$ photoproduction in the photon-induced reaction in the framework of multisource thermal model. The photoproduction of $\eta$ mesons from nucleons can provide valuable information about the excitation spectrum of the nucleons. The model results are in good agreement with the experimental data from $\eta \longrightarrow 3 \pi^{0} \longrightarrow 6 \gamma$ decay mode. It is shown that the movement factor increases linearly with the photon beam energies. The deformation and translation of emission sources are visually given in the formalism.

This issue brings together a collection of articles on properties of chemical and kinetic freeze-outs in high energy nuclear collisions. We hope this will be a useful issue for researchers working in related areas. Meanwhile, we regret that more manuscripts submitted for publication in this issue have not been accepted according to reviewer's reports.

\section{Conflicts of Interest}

The authors declare that they have no conflicts of interest or private agreements with companies.

\section{Acknowledgments}

We sincerely thank all the authors who contributed to this issue and all the reviewers who reviewed to this issue.

$\mathrm{Fu}-\mathrm{Hu} \mathrm{Liu}$

Sakina Fakhraddin

Roy A. Lacey

Raghunath Sahoo

Edward K. Sarkisyan-Grinbaum

Bhartendu K. Singh 

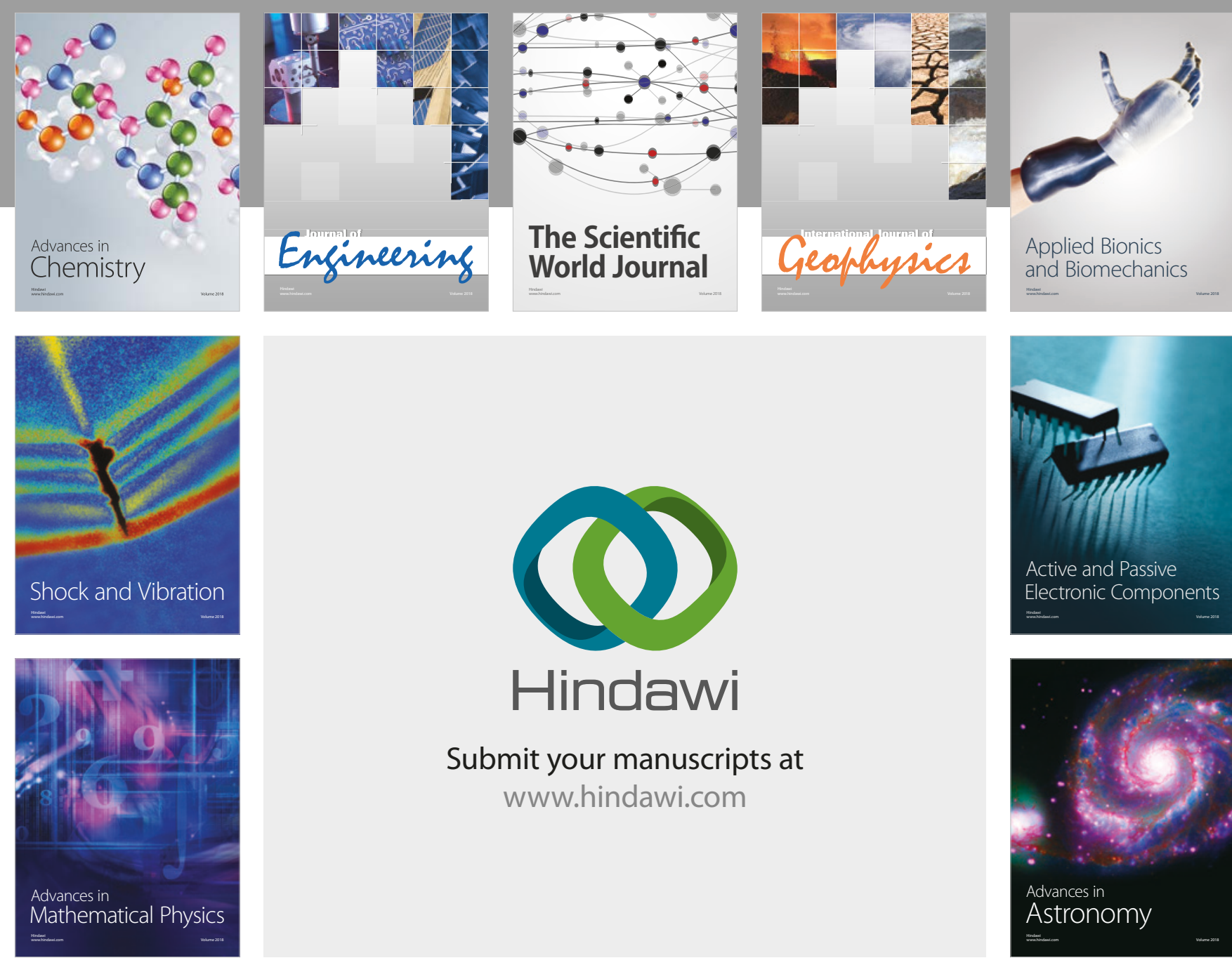

Submit your manuscripts at

www.hindawi.com

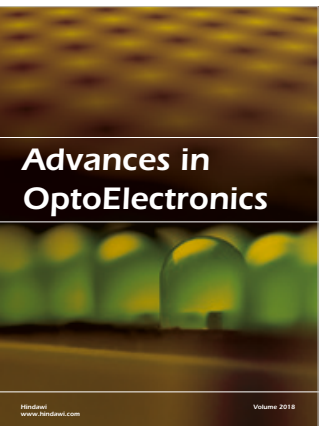

\section{Rotcting Machinery}
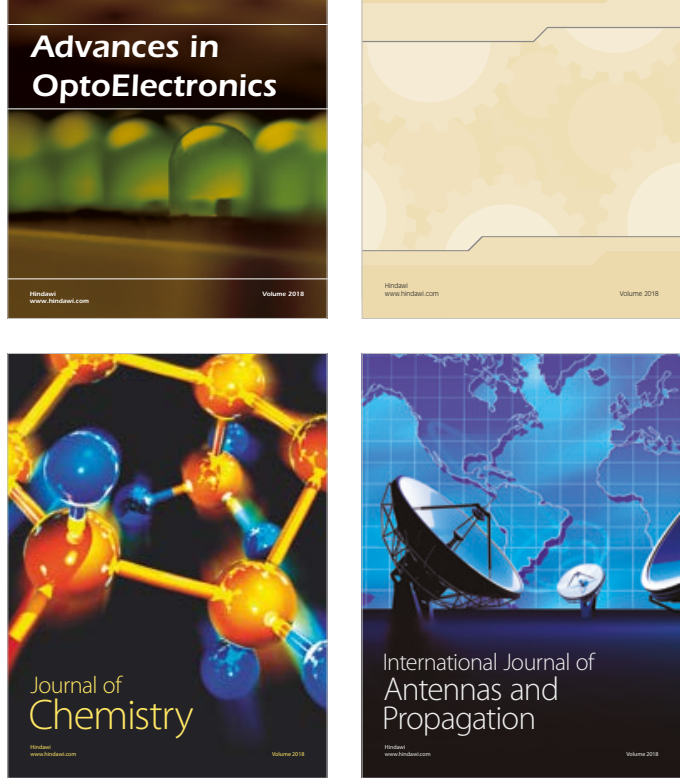

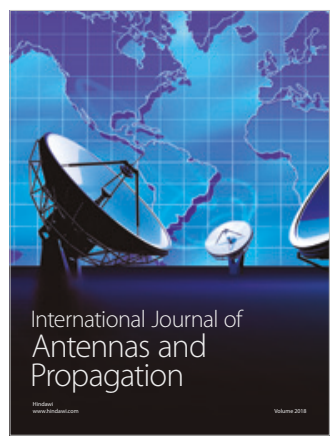

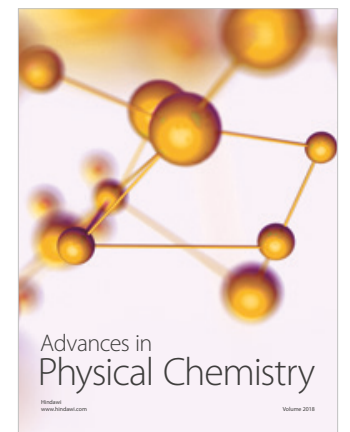

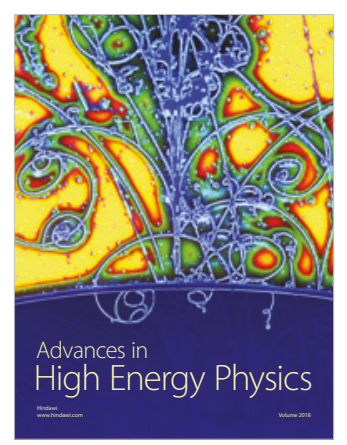

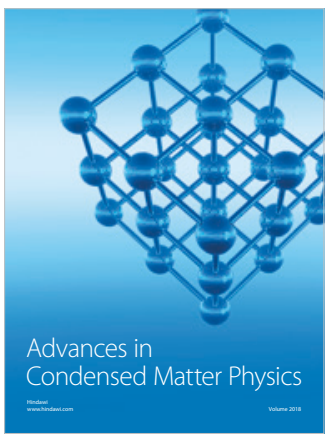

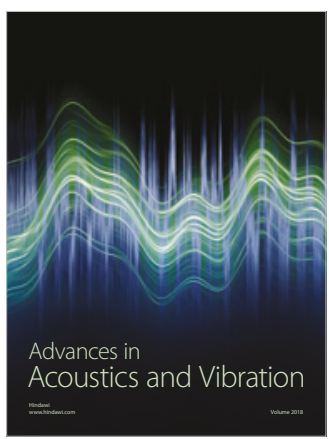

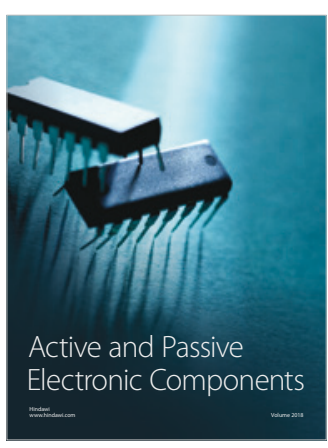
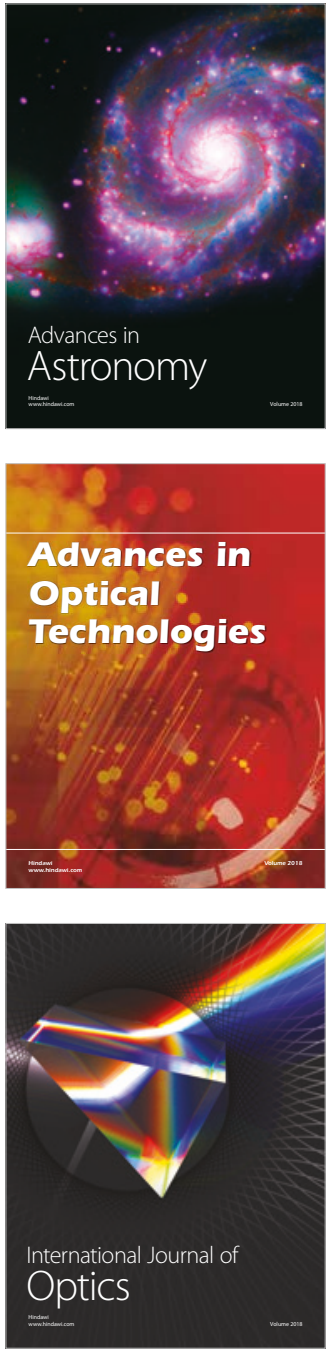